\title{
Of best practice in catalysis
}

Reporting data according to standards accepted by the community is fundamental to the progress of science. In the broad area of catalysis, best practice principles are well consolidated in some subfields, but they are still developing in others.

S cientific disciplines are organized according to specific paradigms, to use a concept popularized by Thomas Kuhn in The Structure of Scientific Revolutions ${ }^{1}$. From such paradigms, coherent systems of rules can be derived, which eventually guide the scientific endeavour of each single scientist. Going beyond their philosophical dimension, such rules translate into those set of procedures and validated methodologies that are often regarded by researchers as best practices in a field.

Scientific journals are constantly confronted with the task of ensuring data reporting according to best practice procedures, in order to improve reproducibility and facilitate peer review. For instance, in the area of photovoltaics Nature Research journals have introduced, upon discussion with relevant experts, a specific author checklist ${ }^{2}$. In this way, key technical and procedural information relating to the characterization of photovoltaic devices are systematically included in each relevant publication. Such initiatives can perhaps be perceived by some as too intrusive. However, it can be argued that the benefits for the community compensate for the burden. Luckily, checklists and stringent submission requirements are not always necessary, while the discussion about best practice principles can occasionally be revived and presented to the relevant community in the form of comments, perspectives, reviews and even research articles.

\section{"The discussion about best}

\section{practice principles can}

occasionally be revived and presented to the relevant community in the form of comments, perspectives,

\section{reviews and even research articles:"}

Inspired by the spread of their incoming submissions, several journals that consider manuscripts in the area of catalysis have recently provided specific guidelines for catalysis researchers, which detail for instance the proper information required to determine the stability of a heterogeneous catalyst $^{3}$, or the correct approach to calculate turnover numbers and turnover frequencies ${ }^{4}$. As catalysis grows as a multidisciplinary area of research, such kinds of tutorial publications represent a valuable and handy resource, especially for those who are less familiar with the standard reporting practices in the field.

In line with the experience of other catalysis journals, we at Nature Catalysis have observed in the past three years a broad range of submissions that occasionally contain methodological flaws relating to the characterization of a catalytic process. Here, we do not intend to provide a comprehensive list of such common pitfalls, but rather to raise awareness of the problem. As such, we thought to recall a few representative examples among the most frequent, to inform new potential authors about those typical shortcomings that represent a major obstacle towards publication.

Heterogeneous catalysis is often dealing with processes of direct industrial relevance. In such cases, the ability to produce large product volumes is crucial and this translates into the requirement for long catalyst lifetimes - usually months - in order to reduce costs ${ }^{5}$. Catalyst stability - intended as its ability to retain catalytic function over time, rather than as structural stability - is routinely assessed through time-on-stream or recycling experiments. However, such tests are occasionally performed with reactions run at full conversion ${ }^{6}$. In these cases, however, the reactions are limited by the amount of available reagent and a potential activity decrease won't be revealed. As those conditions do not reflect a kinetically controlled regime, any stability claim remains unsupported. In this case, best practice suggests performing experiments at low conversion, or away from equilibrium conversion.

The area of photocatalysis is also characterized by a recurring erroneous practice when it comes to the determination of activity. In fact, rates - expressed in the form of $\mathrm{mol} \mathrm{g}^{-1} \mathrm{~h}^{-1}$ or similar - are frequently provided as means to compare the performance of different particulate photocatalysts or to claim advances over previous literature. However, as it has been already discussed earlier ${ }^{7}$, the role of the photons contributing to the reaction must be accounted for, while rates are not consistently proportional to the photocatalysts' concentration. It follows that the apparent quantum yields must always be included to appropriately report the activity of a photocatalyst.

While several areas have established and consolidated their metrics and best practice principles, one should not forget that the broader field of catalysis encompasses a diversity of subfields in continuous development. In some cases, the basic requirements necessary to describe a catalytic process may need revision as new knowledge is generated. In this regard, a recent example comes from the electrochemical reduction of nitrogen to ammonia. The production of ammonia via the Haber-Bosch process entails a large burden in terms of energy consumption and carbon footprint. Its electrochemical counterpart, powered by renewable electricity, hence holds great promise to replace an energy and carbon intensive synthesis with a greener alternative. However, current electrocatalytic strategies barely afford production in the parts-per-million range, and real rates are often inflated by ammonia produced from impurities - such as $\mathrm{NO}_{x}$ - or even $\mathrm{NH}_{3}$ directly present in the electrolyte, catalyst, membrane, gas feed or eventually in ambient air. The research community has been striving to ensure the reliable quantification of ammonia from the catalytic process and a series of protocols have recently been developed ${ }^{8,9}$.

More and more examples could be provided at this point. However, as mentioned above, this is not the purpose of this Editorial. We hope instead that these considerations may help to invigorate the discussion on best practice in catalysis and contribute to the consolidation of data reporting in the field.

Published online: 18 June 2020 https://doi.org/10.1038/s41929-020-0480-7

References

1. Kuhn, T. S. The Structure of Scientific Revolutions 4 th edn (Univ. of Chicago Press, 2012). 
2. Nat. Mater. 14, 1073 (2015).

3. Scott, S. L. ACS Catal. 8, 8597-8599 (2018).

4. Schüth, F., Ward, M. D. \& Buriak, J. M. Chem. Mater. 30(11), 3599-3600 (2018).
5. Lange, J.-P. Catal. Sci. Technol. 6 ,

4759-4767 (2016).

6. Kramm, U. I., Marschall, R. \& Rose, M. Chem CatChem 11, 2563-2574 (2019)
7. Kisch., H. Angew. Chem. Int. Ed. 52, 812-847 (2013).

8. Andersen, S. Z. et al. Nature 570, 504-508 (2019).

9. Suryanto, B. H. R. et al. Nat. Catal. 2, 290-296 (2019). 